\title{
Cubic graphs with small independence ratio
}

\author{
József Balogh* \\ Department of Mathematics \\ University of Illinois at Urbana-Champaign, IL, USA \\ jobal@illinois.edu \\ Alexandr Kostochka ${ }^{\dagger}$ \\ Department of Mathematics \\ University of Illinois at Urbana-Champaign, IL, USA \\ Sobolev Institute of Mathematics, Novosibirsk 630090, Russia \\ kostochk@math.uiuc.edu \\ Xujun Liu ${ }^{\ddagger}$ \\ Department of Mathematics \\ University of Illinois at Urbana-Champaign, IL, USA \\ xliu150@illinois.edu \\ Submitted: Aug 30, 2017; Accepted: Feb 11, 2019; Published: Mar 22, 2019 \\ (C) The authors. Released under the CC BY-ND license (International 4.0).
}

\begin{abstract}
Let $i(r, g)$ denote the infimum of the ratio $\frac{\alpha(G)}{|V(G)|}$ over the $r$-regular graphs of girth at least $g$, where $\alpha(G)$ is the independence number of $G$, and let $i(r, \infty):=$ $\lim _{g \rightarrow \infty} i(r, g)$. Recently, several new lower bounds of $i(3, \infty)$ were obtained. In particular, Hoppen and Wormald showed in 2015 that $i(3, \infty) \geqslant 0.4375$, and Csóka improved it to $i(3, \infty) \geqslant 0.44533$ in 2016. Bollobás proved the upper bound $i(3, \infty)<\frac{6}{13}$ in 1981 , and McKay improved it to $i(3, \infty)<0.45537$ in 1987 . There were no improvements since then. In this paper, we improve the upper bound to $i(3, \infty) \leqslant 0.454$.
\end{abstract}

Mathematics Subject Classifications: 05C15, 05C35

*Research of this author is partially supported by NSF Grants DMS-1500121, DMS-1764123, Arnold O. Beckman Research Award (UIUC) Campus Research Board 18132 and the Langan Scholar Fund (UIUC).

${ }^{\dagger}$ Research of this author is supported in part by NSF grant DMS-1600592, by Award RB17164 of the UIUC Campus Research Board, and by grants 18-01-00353 and 19-01-00682 of the Russian Foundation for Basic Research.

${ }^{\ddagger}$ Research of this author is supported in part by Award RB17164 of the Research Board of the University of Illinois at Urbana-Champaign. 


\section{Introduction}

A set $S$ of vertices in a graph $G$ is independent if no two vertices of $S$ are joined by an edge. The independence number, $\alpha(G)$, is the maximum size of an independent set in $G$. The independence ratio, $i(G)$, of a graph $G$ is the ratio $\frac{\alpha(G)}{|V(G)|}$. For positive integers $r$ and $g, i(r, g)$ denotes the infimum of $i(G)$ over the $r$-regular graphs of girth at least $g$, and $i(r, \infty)$ denotes $\lim _{g \rightarrow \infty} i(r, g)$. The first interesting upper bounds on $i(r, \infty)$ were obtained by Bollobás [2] in 1981. In particular, he proved $i(3, \infty)<\frac{6}{13}$. Refining the method, McKay [12] in 1987 showed

Theorem 1 (McKay [12]).

$$
i(3, \infty)<0.45537
$$

In the next 30 years, there were no improvements of Theorem 1, but recently some interesting lower bounds on $i(r, \infty)$ and in particular on $i(3, \infty)$ were proved. Hoppen [7] showed $i(3, \infty) \geqslant 0.4328$. Then Kardoš, Král and Volec [10] improved the bound to 0.4352. Csóka, Gerencsér, Harangi, and Virág [5] pushed the bound to 0.4361 and Hoppen and Wormald [8] — to 0.4375. Moreover, Csóka et al [5] claimed a computer assisted lower bound $i(3, \infty) \geqslant 0.438$, and Csóka [4] later improved the bound to 0.44533. The lower bound of $i(3, \infty)$ was also studied in [6], [11] and [13]. Our result is an improvement of $(1)$ to $i(3, \infty) \leqslant 0.454$. The improvement is small, but it decreases the gap between the upper and lower bounds on $i(3, \infty)$ by approximately $14 \%$.

Theorem 2. $i(3, \infty) \leqslant 0.454$.

The proof uses the language of configurations introduced by Bollobás [3], and shows that "many" 3-regular configurations have "small" independence ratio. The proof of our improvement is based on analyzing the presence not of largest independent sets, but of larger structures, so called MAI-sets (defined in Section 3) that contain largest independent sets.

\section{Preliminaries}

\section{$2.1 \quad$ Notation}

We mostly use standard notation. The complete $n$-vertex graph is denoted by $K_{n}$. If $G$ is a multigraph and $v, u \in V(G)$, then $E_{G}(v, u)$ denotes the set of all edges in $G$ connecting $v$ and $u, e_{G}(v, u):=\left|E_{G}(v, u)\right|$, and $\operatorname{deg}_{G}(v):=\sum_{u \in V(G) \backslash\{v\}} e_{G}(v, u)$. By $\Delta(G)$ we denote the maximum degree of $G$, and by $g(G)$ - the girth (the length of a shortest cycle) of $G$. For $A \subseteq V(G), G[A]$ denotes the submultigraph of $G$ induced by $A$. For $k \in \mathbb{Z}_{>0},[k]$ denotes the set $\{1, \ldots, k\}$.

\subsection{The Configuration Model}

The configuration model in different versions is due to Bender and Canfield [1] and Bollobás [3]. Our work is based on the version of Bollobás. Let $n$ be an even positive 
integer and $V_{n}=[n]$. Consider the Cartesian product $W_{n}=V_{n} \times[3]$. A configuration/pairing (of order $n$ and degree 3 ) is a perfect matching on the vertex set $W_{n}$. There are $(3 n-1) \cdot(3 n-3) \cdot \ldots \cdot 1=(3 n-1) !$ ! such matchings.

Let $\mathcal{F}_{3}(n)$ denote the collection of all $(3 n-1)$ !! possible pairings on $W_{n}$. We project each pairing $F \in \mathcal{F}_{3}(n)$ to a multigraph $\pi(F)$ on the vertex set $V_{n}$ by ignoring the second coordinate. Then $\pi(F)$ is a 3-regular multigraph (which may or may not contain loops and/or multiple edges). Let $\pi\left(\mathcal{F}_{3}(n)\right)=\left\{\pi(F): F \in \mathcal{F}_{3}(n)\right\}$ be the set of 3-regular multigraphs on $V_{n}$. By definition,

each simple graph $G \in \pi\left(\mathcal{F}_{3}(n)\right)$ corresponds to $(3 !)^{n}$ distinct pairings in $\mathcal{F}_{3}(n)$.

We will call the elements of $V_{n}$ - vertices, and of $W_{n}$ - points.

Definition 3. Let $\mathcal{G}_{g}(n)$ be the set of all cubic graphs with vertex set $V_{n}=[n]$ and girth at least $g$ and $\mathcal{G}_{g}^{\prime}(n)=\left\{F \in \mathcal{F}_{3}(n): \pi(F) \in \mathcal{G}_{g}(n)\right\}$.

We will heavily use the following result:

Theorem 4 (Wormald [14], Bollobás [3]). For each fixed $g \geqslant 3$,

$$
\lim _{n \rightarrow \infty} \frac{\left|\mathcal{G}_{g}^{\prime}(n)\right|}{\left|\mathcal{F}_{3}(n)\right|}=\exp \left\{-\sum_{k=1}^{g-1} \frac{2^{k-1}}{k}\right\} .
$$

Remark. When we say that a pairing $F$ has a multigraph property $\mathcal{A}$, we mean that $\pi(F)$ has property $\mathcal{A}$.

Since dealing with pairings is simpler than working with labeled simple regular graphs, we need the following well-known consequence of Theorem 4.

Corollary 5 ([12](Corollary 1.1), [9](Theorem 9.5)). For fixed $g \geqslant 3$, any property that holds for $\pi(F)$ for almost all pairings $F \in \mathcal{F}_{3}(n)$ also holds for almost all graphs in $\mathcal{G}_{g}(n)$.

Definition 6. For a graph $G$, let $I(G)$ denote the total number of all independent sets in $G$, including the empty set. For integers $r \geqslant 0, g \geqslant 3$, we define

$$
I(r, g)=\inf I(G)^{1 /|V(G)|},
$$

where the infimum is over all graphs $G$ of maximum degree at most $r$ and girth at least $g$.

Recall that the Fibonacci numbers $F_{n}$ are defined by $F_{1}=F_{2}=1$, and $F_{i}=F_{i-1}+F_{i-2}$, for $i \geqslant 3$. The exact formula for $F_{i}$ is

$$
F_{i}=\frac{\varphi^{i}-\psi^{i}}{\sqrt{5}}
$$

where $i \geqslant 0, \varphi=\frac{1+\sqrt{5}}{2}$, and $\psi=\frac{1-\sqrt{5}}{2}$.

Lemma 7 (McKay [12]). For any $g \geqslant 4, I(2, g)=\left(F_{s-1}+F_{s+1}\right)^{\frac{1}{s}}$, where $s=2\lfloor g / 2\rfloor+1$. 
Remark 8. The numbers $s-1$ and $s+1$ in Lemma 7 are even. Therefore,

$$
\begin{aligned}
I(2, g) & =\left(F_{s-1}+F_{s+1}\right)^{1 / s}=\left(\frac{\varphi^{s-1}+\varphi^{s+1}-\varphi^{1-s}-\varphi^{-s-1}}{\sqrt{5}}\right)^{1 / s} \\
& =\varphi \cdot\left(\left(1-\varphi^{-2 s}\right) \frac{\varphi^{-1}+\varphi}{\sqrt{5}}\right)^{1 / s}=\varphi\left(1-\varphi^{-2 s}\right)^{1 / s} .
\end{aligned}
$$

Since the function $\left(1-\varphi^{-2 s}\right)^{1 / s}$ monotonically increases for $s \geqslant 1$, and $\varphi\left(1-\varphi^{-18}\right)^{1 / 9} \geqslant$ 1.618002, we conclude that for each graph $H$ with maximum degree at most 2 and girth at least 8 ,

$$
1.618 \leqslant I(2,8) \leqslant I(H)^{1 /|V(H)|} .
$$

\section{MAI sets in cubic graphs}

Definition 9. A vertex set $A$ in a graph $G$ is an $A I$ set (an almost independent set), if every component of $G[A]$ is an edge or an isolated vertex. In other words, $A$ is an $A I$ set if $\Delta(G[A]) \leqslant 1$.

Definition 10. A vertex set $A$ is a maximum almost independent set (MAI set) in a graph $G$ if all of the following hold:

M1. $A$ is an AI set;

M2. $A$ contains an independent set $A^{\prime}$ of size $\alpha(G)$;

M3. $A$ is largest among all sets satisfying M1 and M2.

Let $G \in \mathcal{G}_{16}(n)$ and $A$ be a MAI set. Denote $B=V(G)-A$.

Lemma 11. $B$ is an $A I$ set.

Proof. Let $b \in B$. We prove that $d_{G[B]}(b) \leqslant 1$. Let $A^{\prime}$ be a maximum independent set in $A$. If $d_{G[B]}(b)=3$, then there is no edge from $b$ to $A$, and $A^{\prime} \cup\{b\}$ is an independent set in $G$ with size $\left|A^{\prime}\right|+1=\alpha(G)+1$, contradicting the definition of $\alpha(G)$.

If $d_{G[B]}(b)=2$, then there is only one edge $e$ from $b$ to $A$, say $b a$. If $d_{G[A]}(a)=0$, then $G[A \cup\{b\}]$ is an AI set in $G$ larger than $A$ containing $A^{\prime}$. This contradicts the fact that $A$ is a MAI set. If $d_{G[A]}(a)=1$, then without loss of generality, we may assume $a \in A-A^{\prime}$. Then $b$ has no neighbors in $A^{\prime}$, and $A^{\prime} \cup\{b\}$ is an independent set in $G$ with size $\left|A^{\prime}\right|+1$, again contradicting the definition of $\alpha(G)$.

Let $A$ be a MAI set in $G \in G_{16}(n)$. Denote the set of vertices with degree 1 in $G[A]$ by $Y$, the set of vertices with degree 1 in $G[B]$ by $Z$. We introduce notation for the sizes of the sets: Let $x:=\left|A^{\prime}\right|, s:=|Y| / 2, t:=|Z| / 2$, and $i:=\frac{n}{2}-|A|$. Then $|A|=\frac{n}{2}-i$ and $|B|=\frac{n}{2}+i$.

Lemma 12. $i \geqslant 0$ and $t \geqslant s$. 
Proof. We count the number of edges with one end in $A$ and one end in $B$ in two ways. We have

$$
2 s \cdot 2+\left(\frac{n}{2}-i-2 s\right) \cdot 3=e[A, B]=2 t \cdot 2+\left(\frac{n}{2}+i-2 t\right) \cdot 3,
$$

i.e.,

$$
t-s=3 i
$$

We also know that $x=\alpha(G)$, so

$$
x=\frac{n}{2}-i-s \geqslant \frac{n}{2}+i-t,
$$

i.e.,

$$
2 i \leqslant t-s=3 i
$$

which implies that

$$
i \geqslant 0 \text { and } t \geqslant s .
$$

Lemma 13. If $G \in \mathcal{G}_{5}(n)$, then

(i) each vertex in $Z$ has degree at most one to $Y$;

(ii) each vertex in $Y$ has degree at most one to $Z$.

Proof. (i) Suppose $z \in Z$ and $N_{G}(z)=\left\{z^{\prime}, y_{1}, y_{2}\right\}$, where $z^{\prime} \in Z$ and $y_{1}, y_{2} \in Y$. Since $g(G) \geqslant 4, y_{1} \neq y_{2}, y_{1} y_{2} \notin E(G)$, and so $A-y_{1}-y_{2}$ contains an independent set $A^{\prime}$ with $\left|A^{\prime}\right|=\alpha(G)$. Thus the set $A^{\prime}+z$ is an independent set of size $\alpha(G)+1$ contradicting the definition of $\alpha(G)$.

(ii) Similarly, suppose $y \in Y$ and $N_{G}(y)=\left\{y^{\prime}, z_{1}, z_{2}\right\}$, where $y^{\prime} \in Y$ and $z_{1}, z_{2} \in$ $Z$. Then $A-y$ contains an independent set $A^{\prime}$ with $\left|A^{\prime}\right|=\alpha(G)$. For $i=1,2$, let $N_{G}\left(z_{i}\right)=\left\{z_{i}^{\prime}, y, a_{i}\right\}$, where $z_{i}^{\prime} \in Z$. By Part (i), $a_{1}, a_{2} \notin Y$. Since $g(G) \geqslant 5, a_{2} \neq a_{1}$. Then $(A-y) \cup\left\{z_{1}, z_{2}\right\}$ is an AI set containing $A^{\prime}$ and is larger than $A$, a contradiction.

Let $J=\left\{y_{1} z_{1}, \ldots, y_{j} z_{j}\right\}$ be the set of all edges connecting $Y$ with $Z$ in $G$. By Lemma $13, J$ is a matching in $G$. Define an auxiliary graph $H=H(A)$ as follows: $V(H)=J$, and $y_{\ell} z_{\ell}$ is adjacent to $y_{\ell^{\prime}} z_{\ell^{\prime}}$ if $y_{\ell} y_{\ell^{\prime}} \in E(G)$ or $z_{\ell} z_{\ell^{\prime}} \in E(G)$. By construction, the maximum degree of $H$ is at most 2 and a cycle of length $c$ in $H$ corresponds to a cycle of length $2 c$ in $G$.

Lemma 14. The graph $G$ contains at least $I(H)$ distinct MAIs.

Proof. Let $J^{\prime}=\left\{y_{1} z_{1}, \ldots, y_{j^{\prime}} z_{j^{\prime}}\right\}$ be an arbitrary independent set in $H$. Then the sets $Y_{1}=\left\{y_{1}, \ldots, y_{j^{\prime}}\right\}$ and $Z_{1}=\left\{z_{1}, \ldots, z_{j^{\prime}}\right\}$ are independent in $G$. By the definition of $Y$, $A-Y_{1}$ contains an independent set $A^{\prime}$ with $\left|A^{\prime}\right|=\alpha(G)$. Let $A_{1}=\left(A-Y_{1}\right) \cup Z_{1}$. By Lemma 13 , the degree in $G\left[A_{1}\right]$ of every vertex in $\left(Y-Y_{1}\right) \cup Z_{1}$ is at most 1 . If a vertex $a \in A-Y$ is adjacent to two vertices, say $z_{1}, z_{2}$ in $Z_{1}$, then the set $\left(A^{\prime}-a\right) \cup\left\{z_{1}, z_{2}\right\}$ is independent and is larger than $A^{\prime}$, a contradiction. Thus, $A_{1}$ is an AI set. Since $\left|A_{1}\right|=|A|$, this proves the lemma.

Remark 15. Recall that $|A|=\frac{n}{2}-i,|B|=\frac{n}{2}+i,|Y|=2 s=2\left(\frac{n}{2}-i-x\right)$, and $|A-Y|=2 x-\frac{n}{2}+i$. By (6), we know that $t=3 i+s=\frac{n}{2}+2 i-x$. Therefore, $|Z|=2 t=2\left(\frac{n}{2}+2 i-x\right)$ and $|B-Z|=2 x-\frac{n}{2}-3 i$. By $(5), e[A, B]=2 x+\frac{n}{2}-i$. 

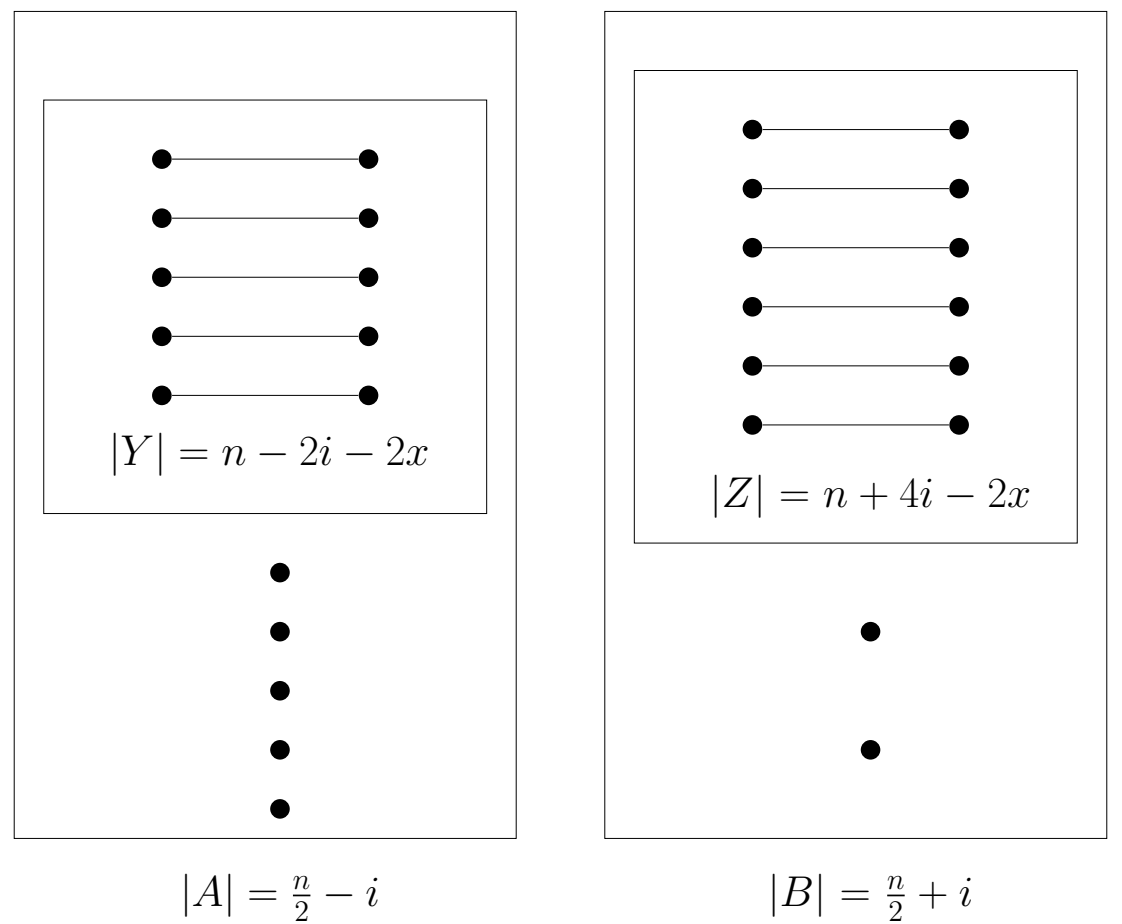

Figure 1: A MAI set $A$.

\section{The set up of the proof}

\subsection{Restating the theorem}

We will use Theorem 1 of McKay in the following stronger form.

Theorem 16 (McKay [12]). For every $\epsilon>0$, there exists an $N>0$ such that for each $n>N$,

$$
\left|\left\{F \mid F \in \mathcal{F}_{3}(n): \alpha(\pi(F))>0.45537 n\right\}\right|<\epsilon \cdot(3 n-1) ! !
$$

We will show that "almost all" cubic labeled graphs of girth at least 16 have independence ratio at most 0.454 . In view of Theorem 4 , the following more technical statement implies Theorem 2.

Theorem 17. For every $\epsilon>0$, there is an $N>0$ such that for each $n>N$,

$$
\left|\left\{F \in \mathcal{G}_{16}^{\prime}(n): \alpha(\pi(F))>0.454 n\right\}\right|<\epsilon(3 n-1) ! !
$$

A referee asked whether one can derive from Theorem 17 that a random cubic graph $G \in G_{n, 3}$ asymptotically almost surely satisfies $\alpha(G) \leqslant 0.454 n$. We do not see how to derive this from the statement of the theorem but think that one can modify our proof to show this fact.

The rest of the paper is a proof of Theorem 17. By definition, every graph has a MAI set. So, for large $n$, nonnegative integers $x \geqslant 0.454 n$ and $i \leqslant \frac{n}{2}-x$, and each set $A$ of size $\frac{n}{2}-i$ with a fixed matching of size $\frac{n}{2}-i-x$ we will estimate the total $x$-weight of configurations $F \in \mathcal{G}_{16}^{\prime}(n)$ in which $A$ forms a MAI set. The idea of the weight (used by McKay in [12]) is to decrease overcount of the configurations containing a given MAI set, 
but guarantee that the total weight of each configuration containing at least one MAI set with independence number $x$ would be at least 1 .

\subsection{Setup of the proof of Theorem 17}

An $A I$-pair on $[n]$ is a pair $(A, R)$ consisting of a set $A \subset[n]$ and a matching $R$ on a subset of $A$ such that $E(G[A])=R$. The independence number, $\alpha(A, R)$, of an AI-pair $(A, R)$ is $|A|-|R|$. Let $\mathcal{P}(n, x)$ denote the family of all AI-pairs $(A, R)$ on $[n]$ with $\alpha(A, R)=x$.

A preimage of an AI-pair $(A, R)$ on $[n]$ is a pair $(\hat{A}, \hat{R})$ where $\hat{A}=A \times[3]$ and $\hat{R}$ is a matching on a subset of $\hat{A}$ with $|\hat{R}|=|R|$ such that for each edge $(i, j)\left(i^{\prime}, j^{\prime}\right) \in \hat{R}$, $i i^{\prime} \in R$. In other words, each edge $e \in R$ is obtained from an edge in $\hat{e} \in \hat{R}$ by ignoring the second coordinates of the ends of $\hat{e}$, and this mapping is one-to-one.

By the $x$-weight of a configuration $F$ we mean

$\omega_{x}(F):=$ the reciprocal of the number of preimages $(\hat{A}, \hat{R}) \subseteq F$ of AI-pairs $(A, R)$ on $[n]$ such that $A$ is an AI set in $\pi(F)$ with $E(\pi(F)[A])=R$ and $\alpha(A, R)=x$.

By the definition of $x$-weight, each pairing $F \in \mathcal{G}_{16}^{\prime}(n)$ with $\alpha(\pi(F))=x$ contributes exactly 1 to

$$
\begin{gathered}
\sigma(n, x, 16):= \\
\sum_{(A, R) \in \mathcal{P}(n, x)}\left\{\omega_{x}\left(F^{\prime}\right): F^{\prime} \in \mathcal{G}_{16}^{\prime}(n) \text { and }(\hat{A}, \hat{R}) \text { is an induced subpairing of } F^{\prime}\right\} .
\end{gathered}
$$

It follows that

$$
\sigma(n, x, 16) \geqslant \mid\left\{F^{\prime} \in \mathcal{G}_{16}^{\prime}(n) \text { with } \alpha\left(\pi\left(F^{\prime}\right)\right)=x\right\} \mid .
$$

Lemma 18. Let $n$ be a positive even integer and $x$ be an integer with $0.454 n<x \leqslant$ $0.45537 n$. The number of pairings $F \in \mathcal{G}_{16}^{\prime}(n)$ such that $\pi(F)$ has a MAI set $A$ with $\left|A^{\prime}\right|=x$ is at most

$$
\begin{aligned}
q(x, n):= & \sum_{i=0}^{\frac{n}{2}-x}\left(\begin{array}{c}
n \\
\frac{n}{2}-i
\end{array}\right) \cdot \frac{\left(\frac{n}{2}-i\right) ! \cdot 3^{(n-2 x-2 i)}}{\left(2 x+i-\frac{n}{2}\right) ! \cdot 2^{\frac{n}{2}-x-i} \cdot\left(\frac{n}{2}-x-i\right) !} \\
& \cdot \frac{\left(\frac{n}{2}+i\right) ! \cdot 3^{n-2 x+4 i}}{\left(2 x-3 i-\frac{n}{2}\right) ! \cdot 2^{\frac{n}{2}-x+2 i} \cdot\left(\frac{n}{2}-x+2 i\right) !} \\
& \cdot \sum_{j=0}^{n-2 i-2 x}\left(\begin{array}{c}
n-2 i-2 x \\
j
\end{array}\right) \cdot\left(\begin{array}{c}
n-2 x+4 i \\
j
\end{array}\right) \cdot 2^{2 j} \cdot j ! \cdot\left(\frac{1}{1.618}\right)^{j} \\
& \cdot \frac{\left(3\left(2 x-\frac{n}{2}-3 i\right)\right) ! \cdot\left(3\left(2 x-\frac{n}{2}+i\right)\right) !}{\left(3\left(2 x-\frac{n}{2}-3 i\right)-2(n-2 i-2 x)+j\right) !}
\end{aligned}
$$

Proof. By (10), it is enough to show that $\sigma(n, x, 16) \leqslant q(x, n)$. Below we describe a procedure of constructing for every AI-pair $(A, R)$ on $[n]$ with $\alpha(A, R)=x$ all pairings in $F \in \mathcal{G}_{16}^{\prime}(n)$ for which $A$ is a MAI set. Not every obtained pairing will be in $\mathcal{G}_{16}^{\prime}(n)$ and some pairings will have independence number larger than $x$, but every $F \in \mathcal{G}_{16}^{\prime}(n)$ such that $A$ is a MAI set in $\pi(F)$ will be a result of this procedure. 
0 . Choose nonnegative integers $n, x, i, j$ such that $n$ is even, $0.454 n<x \leqslant 0.45537 n$, $i \leqslant \frac{n}{2}-x$, and $j \leqslant \frac{n}{2}-x-i$.

1. Choose a set $A \subset[n]$ with $|A|=\frac{n}{2}-i$. There are $\left(\begin{array}{c}n \\ \frac{n}{2}-i\end{array}\right)$ ways to do it.

2. Choose a matching $R$ on $A$ with $|R|=\frac{n}{2}-x-i$. There are

$$
\frac{\left(\frac{n}{2}-i\right) !}{\left(2 x+i-\frac{n}{2}\right) ! \cdot 2^{\frac{n}{2}-x-i} \cdot\left(\frac{n}{2}-x-i\right) !}
$$

ways to do it. Then there are $3^{n-2 x-2 i}$ ways to decide which point of each chosen end of an edge in $R$ will be the end of the corresponding edge in $F$.

3. Similarly to Step 2, we have

$$
\frac{\left(\frac{n}{2}+i\right) !}{\left(2 x-3 i-\frac{n}{2}\right) ! \cdot 2^{\frac{n}{2}-x+2 i} \cdot\left(\frac{n}{2}-x+2 i\right) !}
$$

ways to construct a matching $R^{\prime}$ of $\frac{n}{2}-x+2 i$ edges on $B:=[n]-A$, since $|B|=\frac{n}{2}+i$. After that there are $3^{n-2 x+4 i}$ ways to decide which point of each chosen end of an edge in $R^{\prime}$ will be the end of the corresponding edge in $F$.

4. Let $Y$ (respectively, $Z$ ) be the set of vertices covered by the matching $R$ (respectively, $\left.R^{\prime}\right)$. By Lemma 13, if $A$ is a MAI-set in $\pi(F)$, then the set of edges connecting $Y$ with $Z$ is a matching. If this matching, say $M$ has $j$ edges, then there are $\left(\begin{array}{c}n-2 i-2 x \\ j\end{array}\right)$ ways to choose the set of the ends of $M$ in $Y$ and $\left(\begin{array}{c}n-2 x+4 i \\ j\end{array}\right) j$ ! ways to choose the ends of $M$ in $Z$. Since there are 2 free points left for each vertex in $Y$ and $Z$, we have $2^{2 j}$ ways to choose which point of each vertex in $Y$ and $Z$ to be used to form an edge in $M$.

5. By Lemma 14 each pairing $F \in \mathcal{G}_{16}^{\prime}(n)$ containing a MAI set $A$ with $j$ edges between $Y$ and $Z$ contains at least $I(2,8)^{j}$ distinct MAI sets of the same cardinality. By Lemma $7, I(2,8)^{j} \geqslant 1.618^{j}$. Hence by $(8), \omega_{x}(F) \leqslant 1.618^{-j}$.

6. Now we choose for each remaining free point $p$ from vertices in $Y$ a free point $q$ in a vertex in $B-Z$ and add edge $p q$. There are

$$
\frac{\left(3\left(2 x-\frac{n}{2}-3 i\right)\right) !}{\left(3\left(2 x-\frac{n}{2}-3 i\right)-2(n-2 i-2 x)+j\right) !}
$$

ways to do it.

7. Similarly to Step 6, we choose for each remaining free point $q$ from vertices in $Z$ a free point $p$ in a vertex in $A-Y$ and add edge $p q$. There are

$$
\frac{\left.3\left(2 x-\frac{n}{2}+i\right)\right) !}{\left(3\left(2 x-\frac{n}{2}+i\right)-2(n-2 x+4 i)+j\right) !}
$$

ways to do it. 
8. Finally, there are $3\left(2 x-\frac{n}{2}+i\right)-2(n-2 x+4 i)+j=10 x-\frac{7 n}{2}-5 i+j$ free points left in $A$ and $10 x-\frac{7 n}{2}-5 i+j$ free points left in $B$. We have $\left(10 x-\frac{7 n}{2}-5 i+j\right)$ ! ways to complete a pairing on $W_{n}$.

In the proofs below we will use Stirling's formula: For every $n \geqslant 1$,

$$
\sqrt{2 \pi n}\left(\frac{n}{e}\right)^{n} \leqslant n ! \leqslant \sqrt{2 \pi n}\left(\frac{n}{e}\right)^{n} e^{1 / 12 n}
$$

We will also use the notation $\frac{\partial}{\partial j}$ to denote the partial derivative with respect to $j$. Moreover, we use the domain $x \geqslant 0$ and define $\ln (0)=-\infty$ when we consider $\ln x$.

Lemma 19. Let $n$ be a positive even integer and $x$ be an integer satisfying $0.454 n<x \leqslant$ $0.45537 n$. Let

$$
\Omega=\left\{(\chi, \zeta, \xi): 0.454<\chi \leqslant 0.45537,0 \leqslant \zeta \leqslant \frac{1}{2}-\chi, 0 \leqslant \xi \leqslant 1-2 \chi-2 \zeta\right\} .
$$

Let

$$
\begin{aligned}
f(\chi, \zeta):= & 3^{\frac{1}{2}-4 \chi+2 \zeta} \cdot(1-2 \chi-2 \zeta)^{1-2 \chi-2 \zeta} \cdot(1-2 \chi+4 \zeta)^{1-2 \chi+4 \zeta} \cdot\left(6 \chi-\frac{3}{2}+3 \zeta\right)^{6 \chi-\frac{3}{2}+3 \zeta} \\
& \cdot\left(6 \chi-\frac{3}{2}-9 \zeta\right)^{6 \chi-\frac{3}{2}-9 \zeta} \cdot\left(2 \chi+\zeta-\frac{1}{2}\right)^{-\left(2 \chi+\zeta-\frac{1}{2}\right)} \cdot 2^{-(1-2 \chi+\zeta)} \\
& \cdot\left(\frac{1}{2}-\chi-\zeta\right)^{-\left(\frac{1}{2}-\chi-\zeta\right)} \cdot\left(\frac{1}{2}-\chi+2 \zeta\right)^{-\left(\frac{1}{2}-\chi+2 \zeta\right)} \cdot\left(2 \chi-3 \zeta-\frac{1}{2}\right)^{-\left(2 \chi-3 \zeta-\frac{1}{2}\right)} \\
g(\chi, \zeta, \xi):= & 2^{2 \xi} \cdot\left(\frac{1}{1.618}\right)^{\xi} \cdot \xi^{-\xi} \cdot(1-2 \chi-2 \zeta-\xi)^{-(1-2 \chi-2 \zeta-\xi)} \\
& \cdot(1-2 \chi+4 \zeta-\xi)^{-(1-2 \chi+4 \zeta-\xi)} \cdot\left(-\frac{7}{2}+10 \chi-5 \zeta+\xi\right)^{\frac{7}{2}-10 \chi+5 \zeta-\xi}
\end{aligned}
$$

and

$$
h(\chi, \zeta, \xi):=f(\chi, \zeta) \cdot g(x, \zeta, \xi)
$$

Then

$$
\frac{q(x, n)}{(3 n-1) ! !}=O\left(n^{6}\right) \cdot \max \left\{(h(\chi, \zeta, \xi))^{n}:(\chi, \zeta, \xi) \in \Omega\right\}
$$

Proof. We write $q(x, n)$ as a double sum of $i$ and $j$ and let $r(x, n, i, j)$ be the function inside the double sum of $q(x, n)$, i.e.,

$$
q(x, n)=\sum_{i=0}^{\frac{n}{2}-x} \sum_{j=0}^{n-2 x-2 i} r(x, n, i, j)
$$

Then certainly,

$$
q(x, n) \leqslant n^{2} \cdot \max \left\{r(x, n, i, j): 0 \leqslant i \leqslant \frac{n}{2}-x, 0 \leqslant j \leqslant n-2 x-2 i\right\} .
$$

So, it is enough to estimate $r(x, n, i, j)$. We know that

$r(x, n, i, j)=\frac{n !}{\left(\frac{n}{2}-i\right) ! \cdot\left(\frac{n}{2}+i\right) !} \cdot \frac{\left(\frac{n}{2}-i\right) ! \cdot 3^{n-2 x-2 i}}{\left(2 x+i-\frac{n}{2}\right) ! \cdot 2^{\frac{n}{2}-x-i} \cdot\left(\frac{n}{2}-x-i\right) !}$ 


$$
\begin{aligned}
& \frac{\left(\frac{n}{2}+i\right) ! \cdot 3^{n-2 x+4 i}}{\left(2 x-3 i-\frac{n}{2}\right) ! \cdot 2^{\frac{n}{2}-x+2 i} \cdot\left(\frac{n}{2}-x+2 i\right) !} \cdot \frac{(n-2 i-2 x) !}{j ! \cdot(n-2 i-2 x-j) !} \\
& \cdot \frac{(n-2 x+4 i) !}{j ! \cdot(n-2 x+4 i-j) !} \cdot 2^{2 j} \cdot j ! \cdot\left(\frac{1}{1.618}\right)^{j} \cdot \frac{\left(6 x-\frac{3 n}{2}-9 i\right) ! \cdot\left(6 x-\frac{3 n}{2}+3 i\right) !}{\left(10 x-\frac{7 n}{2}-5 i+j\right) !} .
\end{aligned}
$$

Recall that

$$
(3 n-1) ! ! \geqslant \frac{(3 n) ! !}{3 n} \geqslant \frac{\sqrt{(3 n) !}}{3 n}
$$

Therefore,

$$
\begin{aligned}
\frac{r(x, n, i, j)}{(3 n-1) ! !} \leqslant & \frac{n ! \cdot(3 n)}{((3 n) !)^{\frac{1}{2}}} \cdot \frac{3^{n-2 x-2 i}}{\left(2 x+i-\frac{n}{2}\right) ! \cdot 2^{\frac{n}{2}-x-i} \cdot\left(\frac{n}{2}-x-i\right) !} \\
& \cdot \frac{3^{n-2 x+4 i}}{\left(2 x-3 i-\frac{n}{2}\right) ! \cdot 2^{\frac{n}{2}-x+2 i} \cdot\left(\frac{n}{2}-x+2 i\right) !} \cdot \frac{(n-2 i-2 x) !}{j ! \cdot(n-2 i-2 x-j) !} \\
& \cdot \frac{(n-2 x+4 i) !}{(n-2 x+4 i-j) !} \cdot 2^{2 j} \cdot\left(\frac{1}{1.618}\right)^{j} \cdot \frac{\left(6 x-\frac{3 n}{2}-9 i\right) ! \cdot\left(6 x-\frac{3 n}{2}+3 i\right) !}{\left(10 x-\frac{7 n}{2}-5 i+j\right) !}
\end{aligned}
$$

Introducing new variables $\chi:=\frac{x}{n}, \zeta:=\frac{i}{n}$, and $\xi:=\frac{j}{n}$ and using Stirling's formula (11), we get

$$
\begin{aligned}
\frac{r(x, n, i, j)}{(3 n-1) ! !}= & O\left(n^{4}\right) \cdot\left(\frac{n}{e}\right)^{n} \cdot\left(\frac{n}{e}\right)^{(1-2 \zeta-2 \chi) n} \cdot\left(\frac{n}{e}\right)^{(1-2 \chi+4 \zeta) n} \cdot\left(\frac{n}{e}\right)^{\left(6 \chi-\frac{3}{2}-9 \zeta\right) n} \cdot\left(\frac{n}{e}\right)^{\left(6 \chi-\frac{3}{2}+3 \zeta\right) n} \\
& \cdot\left(\frac{n}{e}\right)^{-\frac{3}{2} n} \cdot\left(\frac{n}{e}\right)^{-\left(2 \chi+\zeta-\frac{1}{2}\right) n} \cdot\left(\frac{n}{e}\right)^{-\left(\frac{1}{2}-\chi-\zeta\right) n} \cdot\left(\frac{n}{e}\right)^{-\left(2 \chi-3 \zeta-\frac{1}{2}\right) n} \cdot\left(\frac{n}{e}\right)^{-\left(\frac{1}{2}-\chi+2 \zeta\right) n} \cdot\left(\frac{n}{e}\right)^{-\xi n} \\
& \cdot\left(\frac{n}{e}\right)^{-(1-2 \zeta-2 \chi-\xi) n} \cdot\left(\frac{n}{e}\right)^{-(1-2 \chi+4 \zeta-\xi) n} \cdot\left(\frac{n}{e}\right)^{-\left(10 \chi-\frac{7}{2}-5 \zeta+\xi\right) n} \cdot(f(\chi, \zeta) \cdot g(\chi, \zeta, \xi))^{n} .
\end{aligned}
$$

Therefore,

$$
\frac{r(x, n, i, j)}{(3 n-1) ! !}=O\left(n^{4}\right) \cdot(h(\chi, \zeta, \xi))^{n}
$$

This proves the lemma.

Recall that the domain of $h(\chi, \zeta, \xi)$ is $\Omega$ defined in (12). Our main goal now is to show that

$$
\max _{(\chi, \zeta, \xi) \in \Omega} h(\chi, \zeta, \xi) \leqslant 0.999983<1 .
$$

We do this in the next section, and then Theorem 17 easily follows.

\section{Proof of (14)}

In order to find the maximum value of $h(\chi, \zeta, \xi)$ for a fixed $\chi$, we will maximize

$$
\ln (h(\chi, \zeta, \xi))
$$

We first find the value of $\xi$ in terms of $\chi$ and $\zeta$ that maximizes $\ln (g(\chi, \zeta, \xi))$. By definition,

$$
\ln (g(\chi, \zeta, \xi))=\xi \ln \left(\frac{4}{1.618}\right)-(\xi \ln (\xi)+(1-2 \zeta-2 \chi-\xi) \ln (1-2 \zeta-2 \chi-\xi)
$$




$$
\left.+(1-2 \chi+4 \zeta-\xi) \ln (1-2 \chi+4 \zeta-\xi)+\left(10 \chi-\frac{7}{2}-5 \zeta+\xi\right) \ln \left(10 \chi-\frac{7}{2}-5 \zeta+\xi\right)\right) .
$$

Hence

$$
\begin{aligned}
\frac{\partial \ln (g(\chi, \zeta, \xi))}{\partial \xi}= & \ln (1-2 \chi-2 \zeta-\xi)+\ln (1-2 \chi+4 \zeta-\xi) \\
& -\ln \left(10 \chi-5 \zeta+\xi-\frac{7}{2}\right)-\ln (\xi)+\ln \left(\frac{4}{1.618}\right) \\
= & \ln \left(\frac{(1-2 \chi-2 \zeta-\xi) \cdot(1-2 \chi+4 \zeta-\xi) \cdot \frac{4}{1.618}}{\xi \cdot\left(10 \chi-5 \zeta+\xi-\frac{7}{2}\right)}\right)
\end{aligned}
$$

In order to solve

$$
\frac{\partial \ln (g(\chi, \zeta, \xi))}{\partial \xi}=0
$$

we solve the equivalent equation

$$
p(\xi):=4 \cdot(1-2 \chi-2 \zeta-\xi) \cdot(1-2 \chi+4 \zeta-\xi)-1.618 \cdot \xi \cdot\left(10 \chi-5 \zeta+\xi-\frac{7}{2}\right)=0,
$$

where $p(\xi)$ has domain $0 \leqslant \xi \leqslant 1-2 \chi-2 \zeta$. By the quadratic formula, the roots are

$$
\xi_{1}=\frac{-b-\sqrt{b^{2}-4 a c}}{2 a} \text { and } \xi_{2}=\frac{-b+\sqrt{b^{2}-4 a c}}{2 a}
$$

where

$$
\begin{aligned}
& a=2.382 \\
& b=-0.18 \chi+0.09 \zeta-2.337 \\
& c=16 \chi^{2}-32 \zeta^{2}-16 \chi+8 \zeta-16 \chi \zeta+4 .
\end{aligned}
$$

Moreover, for fixed $\chi$ and $\zeta$ satisfying $0.454 \leqslant \chi \leqslant 0.45537$ and $\chi+\zeta \leqslant \frac{1}{2}, p(\xi)$ is a parabola opening upward with $\xi_{1} \leqslant 1-2 \chi-2 \zeta \leqslant \xi_{2}$ because $p(1-2 \chi-2 \zeta) \leqslant 0$, and $g(\chi, \zeta, \xi)$ is a continuous function on $\xi$. Therefore, the maximum of $g(\chi, \zeta, \xi)$ can only be attained at $\xi=\xi_{1}$.

Let $g_{1}(\chi, \zeta)=g\left(\chi, \zeta, \xi_{1}(\chi, \zeta)\right)$. For each fixed $\chi$, consider the maximum of

$$
h_{1}(\chi, \zeta):=f(\chi, \zeta) \cdot g_{1}(\chi, \zeta) .
$$

By definition,

$$
\begin{aligned}
\ln \left(h_{1}\right)= & \left(\frac{1}{2}-4 \chi+2 \zeta\right) \ln (3)+(1-2 \chi-2 \zeta) \ln (1-2 \chi-2 \zeta)+(1-2 \chi+4 \zeta) \ln (1-2 \chi+4 \zeta) \\
& +\left(6 \chi-\frac{3}{2}+3 \zeta\right) \ln \left(6 \chi-\frac{3}{2}+3 \zeta\right)+\left(6 \chi-\frac{3}{2}-9 \zeta\right) \ln \left(6 \chi-\frac{3}{2}-9 \zeta\right)+\xi_{1}(\chi, \zeta) \cdot \ln \left(\frac{4}{1.618}\right) \\
& -\left(2 \chi+\zeta-\frac{1}{2}\right) \ln \left(2 \chi+\zeta-\frac{1}{2}\right)-(1-2 \chi+\zeta) \ln (2)-\left(\frac{1}{2}-\chi-\zeta\right) \ln \left(\frac{1}{2}-\chi-\zeta\right) \\
& -\left(\frac{1}{2}-\chi+2 \zeta\right) \ln \left(\frac{1}{2}-\chi+2 \zeta\right)-\left(2 x-3 \zeta-\frac{1}{2}\right) \ln \left(2 \chi-3 \zeta-\frac{1}{2}\right)-\xi_{1}(\chi, \zeta) \cdot \ln \left(\xi_{1}(\chi, \zeta)\right) \\
& -\left(1-2 \zeta-2 \chi-\xi_{1}(\chi, \zeta)\right) \ln \left(1-2 \zeta-2 \chi-\xi_{1}(\chi, \zeta)\right) \\
& -\left(1-2 \chi+4 \zeta-\xi_{1}(\chi, \zeta)\right) \ln \left(1-2 \chi+4 \zeta-\xi_{1}(\chi, \zeta)\right)
\end{aligned}
$$




$$
-\left(10 \chi-\frac{7}{2}-5 \zeta+\xi_{1}(\chi, \zeta)\right) \ln \left(10 \chi-\frac{7}{2}-5 \zeta+\xi_{1}(\chi, \zeta)\right)
$$

and

$$
\begin{aligned}
\frac{\partial \ln \left(h_{1}\right)}{\partial \zeta}= & -4 \ln (3)+\ln (2)-3+2 \ln \left(2 \chi+\zeta-\frac{1}{2}\right)-\ln \left(\frac{1}{2}-\chi-\zeta\right)+2 \ln \left(\frac{1}{2}-\chi+2 \zeta\right) \\
& -6 \ln \left(2 \chi-3 \zeta-\frac{1}{2}\right)+\ln \left(\frac{4}{1.618}\right) \cdot \frac{\partial \xi_{1}(\chi, \zeta)}{\partial \zeta}-\frac{\partial \xi_{1}(\chi, \zeta)}{\partial \zeta} \cdot\left(\ln \left(\xi_{1}(\chi, \zeta)\right)+1\right) \\
& +\left(2+\frac{\partial \xi_{1}(\chi, \zeta)}{\partial \zeta}\right) \cdot\left(\ln \left(1-2 \zeta-2 \chi-\xi_{1}(\chi, \zeta)\right)+1\right) \\
& +\left(\frac{\partial \xi_{1}(\chi, \zeta)}{\partial \zeta}-4\right) \cdot\left(\ln \left(1-2 \chi+4 \zeta-\xi_{1}(\chi, \zeta)\right)+1\right)+\left(5-\frac{\partial \xi_{1}(\chi, \zeta)}{\partial \zeta}\right) \\
& \cdot\left(\ln \left(10 \chi-\frac{7}{2}-5 \zeta+\xi_{1}(\chi, \zeta)\right)+1\right)
\end{aligned}
$$

where

$$
\begin{aligned}
\frac{\partial \xi_{1}(\chi, \zeta)}{\partial \zeta} & =\frac{1}{2 a} \cdot\left(-\frac{\partial b}{\partial \zeta}-\frac{1}{2} \cdot\left(b^{2}-4 a c\right)^{-\frac{1}{2}} \cdot\left(2 b \frac{\partial b}{\partial \zeta}-4 a \frac{\partial c}{\partial \zeta}\right)\right) \\
\frac{\partial b}{\partial \zeta} & =0.09 \\
\frac{\partial c}{\partial \zeta} & =-16 \chi-64 \zeta+8 .
\end{aligned}
$$

Lemma 20. When $\chi=0.454$, the maximum of $h(\chi, \zeta, \xi)$ over $0 \leqslant \zeta \leqslant 0.046$ and $0 \leqslant \xi \leqslant$ $0.092-2 \zeta$ is at most 0.999983 .

Proof. Fix $\chi=0.454$. For $0 \leqslant \zeta \leqslant 0.046$, denote

$$
\xi_{1}^{\prime}(\zeta):=\frac{\partial \xi_{1}(0.454, \zeta)}{\partial \zeta}, \xi_{1}^{\prime \prime}(\zeta):=\frac{\partial^{2} \xi_{1}(0.454, \zeta)}{\partial \zeta^{2}} \text {, and } \xi_{1}(\zeta):=\xi_{1}(0.454, \zeta) .
$$

We have

$$
\begin{aligned}
\frac{\partial \ln \left(h_{1}(0.454, \zeta)\right)}{\partial \zeta}= & -4 \ln (3)+\ln (2)-3+2 \ln (0.408+\zeta)-\ln (0.046-\zeta)+2 \ln (0.046+2 \zeta) \\
- & 6 \ln (0.408-3 \zeta)+\ln \left(\frac{4}{1.618}\right) \cdot \xi_{1}^{\prime}(\zeta)-\xi_{1}^{\prime}(\zeta) \cdot\left(\ln \left(\xi_{1}(\zeta)\right)+1\right)+\left(2+\xi_{1}^{\prime}(\zeta)\right) \\
& \cdot\left(\ln \left(0.092-2 \zeta-\xi_{1}(\zeta)\right)+1\right)+\left(\xi_{1}^{\prime}(\zeta)-4\right) \\
& \cdot\left(\ln \left(0.092+4 \zeta-\xi_{1}(\zeta)\right)+1\right)+\left(5-\xi_{1}^{\prime}(\zeta)\right) \cdot\left(\ln \left(1.04-5 \zeta+\xi_{1}(\zeta)\right)+1\right), \\
\frac{\partial^{2} \ln \left(h_{1}(0.454, \zeta)\right)}{\partial \zeta^{2}}= & \frac{1}{0.046-\zeta}+\frac{4}{0.046+2 \zeta}+\frac{2}{0.408+\zeta}+\frac{18}{0.408-3 \zeta} \\
& +\ln \left(\frac{4}{1.618}\right) \cdot \xi_{1}^{\prime \prime}(\zeta)-\xi_{1}^{\prime \prime}(\zeta) \cdot\left(\ln \left(\xi_{1}(\zeta)\right)+1\right)-\left(\xi_{1}^{\prime}(\zeta)\right)^{2} \cdot \frac{1}{\xi_{1}(\zeta)} \\
& +\xi_{1}^{\prime \prime}(\zeta) \cdot\left(\ln \left(0.092-2 \zeta-\xi_{1}(\zeta)\right)+1\right)-\left(2+\xi_{1}^{\prime}(\zeta)\right)^{2} \cdot \frac{1}{0.092-2 \zeta-\xi_{1}(\zeta)} \\
& +\xi_{1}^{\prime \prime}(\zeta) \cdot\left(\ln \left(0.092+4 \zeta-\xi_{1}(\zeta)\right)+1\right)-\left(\xi_{1}^{\prime}(\zeta)-4\right)^{2} \cdot \frac{1}{0.092+4 \zeta-\xi_{1}(\zeta)} \\
& -\xi_{1}^{\prime \prime}(\zeta) \cdot\left(\ln \left(1.04-5 \zeta+\xi_{1}(\zeta)\right)+1\right)-\left(\xi_{1}^{\prime}(\zeta)-5\right)^{2} \cdot \frac{1}{1.04-5 \zeta+\xi_{1}(\zeta)},
\end{aligned}
$$


where

$$
\xi_{1}^{\prime \prime}(\zeta)=\frac{1}{2 a} \cdot\left(b^{2}-4 a c\right)^{-\frac{1}{2}} \cdot\left(\frac{1}{4} \cdot\left(b^{2}-4 a c\right)^{-1} \cdot\left(2 b \frac{\partial b}{\partial \zeta}-4 a \frac{\partial c}{\partial \zeta}\right)^{2}-\frac{1}{2} \cdot\left(2\left(\frac{\partial b}{\partial \zeta}\right)^{2}+1024 a\right)\right) .
$$

We will show that

$$
\frac{\partial^{2} \ln \left(h_{1}(0.454, \zeta)\right)}{\partial \zeta^{2}}<0, \text { for all } 0 \leqslant \zeta<0.046
$$

This will guarantee that if we find a solution $\zeta_{0} \in[0,0.046)$ of the equation $\frac{\partial \ln \left(h_{1}(0.454, \zeta)\right)}{\partial \zeta}=$ 0 , then the maximum of $h_{1}(0.454, \zeta)$ over $\zeta \in[0,0.046)$ is attained at $\zeta_{0}$.

Claim 21. For each $\zeta \in[0,0.046),-27.336 \leqslant \xi_{1}^{\prime \prime}(\zeta)<-24.822$.

Proof: By (15) and (16), for $\chi=0.454$, the function $\Delta(\zeta):=b^{2}-4 a c$ is quadratic in $\zeta$ with derivative

$$
\Delta^{\prime}(\zeta)=2 b \frac{\partial b}{\partial \zeta}-4 a \frac{\partial c}{\partial \zeta}
$$

which is linear in $\zeta$ and has minimum at $\zeta=0$ and maximum at $\zeta=0.046$. Therefore,

$$
-7.45 \leqslant \Delta^{\prime}(0) \leqslant \Delta^{\prime}(\zeta) \leqslant \Delta^{\prime}(0.046) \leqslant 20.61
$$

for each $\zeta \in[0,0.046)$. Also for such $\zeta$,

$$
\Delta^{\prime \prime}(\zeta)=2\left(\frac{\partial b}{\partial \zeta}\right)^{2}-4 a \frac{\partial^{2} c}{\partial \zeta^{2}}=2 \cdot 0.09^{2}-4 \cdot 2.382 \cdot(-64) \in(609.8,609.81),
$$

so $\Delta(\zeta)$ is a parabola opening upward with minimum attained at the unique root $\zeta_{\xi}$ of the equation $\Delta^{\prime}(\zeta)=0$. By $\Delta^{\prime}(0.012213)<-0.00039, \Delta^{\prime}(0.012214)>0.000219$, and the above statements,

$$
0.012213 \leqslant \zeta_{\xi} \leqslant 0.012214
$$

Hence $\Delta\left(\zeta_{\xi}\right)$ satisfies

$$
\begin{aligned}
5.4821 \leqslant 5.48214-0.0004 \cdot 0.000001 & \leqslant \Delta(0.012213)+\Delta^{\prime}(0.012213) \cdot 0.000001 \\
& \leqslant \Delta\left(\zeta_{\xi}\right) \leqslant \Delta(0.012213) \leqslant 5.4822,
\end{aligned}
$$

and the maximum of $\Delta(\zeta)$ over $\zeta \in[0,0.046)$ is attained at $\zeta=0.046$ and satisfies

$$
5.83019 \leqslant \Delta(0.046) \leqslant 5.8302 .
$$

Therefore, for each $\zeta \in[0,0.046)$,

$$
\begin{aligned}
0.41415 \leqslant \frac{1}{\sqrt{5.8302}} & \leqslant(\Delta(\zeta))^{-\frac{1}{2}} \leqslant \frac{1}{\sqrt{5.4821}} \leqslant 0.427098 \\
0.17152 & \leqslant(\Delta(\zeta))^{-1} \leqslant 0.182412 .
\end{aligned}
$$

Thus by (17),

$$
-27.336 \leqslant \frac{1}{2 \cdot 2.382} \cdot 0.427098 \cdot(0-0.5 \cdot 609.81) \leqslant \xi_{1}^{\prime \prime}(\zeta)
$$




$$
\begin{aligned}
& =\frac{1}{2 a} \cdot(\Delta(\zeta))^{-\frac{1}{2}} \cdot\left(\frac{1}{4} \cdot(\Delta(\zeta))^{-1} \cdot\left(\Delta^{\prime}(\zeta)\right)^{2}-\frac{1}{2} \cdot \Delta^{\prime \prime}(\zeta)\right) \\
& \leqslant \frac{1}{2 \cdot 2.382} \cdot 0.41415 \cdot\left(0.25 \cdot 0.182412 \cdot(20.61)^{2}-0.5 \cdot 609.8\right) \leqslant-24.822
\end{aligned}
$$

This proves Claim 21.

Claim 22. For each $\zeta \in[0,0.046),-0.91445 \leqslant \xi_{1}^{\prime}(0.046) \leqslant \xi_{1}^{\prime}(\zeta) \leqslant \xi_{1}^{\prime}(0) \leqslant 0.31359$.

Proof: By Claim 21, $\xi_{1}^{\prime}(\zeta)$ is a decreasing function on $0 \leqslant \zeta \leqslant 0.046$.

To prove (18), we write $\frac{\partial^{2} \ln \left(h_{1}(0.454, \zeta)\right)}{\partial \zeta^{2}}$ in a form

$$
\frac{\partial^{2} \ln \left(h_{1}(0.454, \zeta)\right)}{\partial \zeta^{2}}=A_{1}(\zeta)+A_{2}(\zeta)+A_{3}(\zeta)+A_{4}(\zeta)+A_{5}(\zeta)
$$

and then bound these expressions separately so that the sum of the upper bounds will be negative for each $\zeta \in[0,0.046)$. By definition

$$
\begin{aligned}
\frac{\partial^{2} \ln \left(h_{1}\right)}{\partial \zeta^{2}}= & \frac{1}{0.046-\zeta}-\xi_{1}^{\prime \prime}(\zeta) \cdot \ln \left(\xi_{1}(\zeta)\right)-\frac{\left(\xi_{1}^{\prime}(\zeta)\right)^{2}}{\xi_{1}(\zeta)}+\frac{4}{0.046+2 \zeta}+\frac{2}{0.408+\zeta} \\
& +\frac{18}{0.408-3 \zeta}+\ln \left(\frac{4}{1.618}\right) \cdot \xi_{1}^{\prime \prime}(\zeta)+\xi_{1}^{\prime \prime}(\zeta) \cdot \ln \left(0.092-2 \zeta-\xi_{1}(\zeta)\right)-\frac{\left(2+\xi_{1}^{\prime}(\zeta)\right)^{2}}{0.092-2 \zeta-\xi_{1}(\zeta)} \\
& +\xi_{1}^{\prime \prime}(\zeta) \cdot \ln \left(0.092+4 \zeta-\xi_{1}(\zeta)\right)-\frac{\left(\xi_{1}^{\prime}(\zeta)-4\right)^{2}}{0.092+4 \zeta-\xi_{1}(\zeta)} \\
& -\xi_{1}^{\prime \prime}(\zeta) \cdot \ln \left(1.04-5 \zeta+\xi_{1}(\zeta)\right)-\frac{\left(\xi_{1}^{\prime}(\zeta)-5\right)^{2}}{1.04-5 \zeta+\xi_{1}(\zeta)}
\end{aligned}
$$

Let

$$
\begin{aligned}
A_{1}(\zeta) & :=\frac{1}{0.046-\zeta}-\left(\xi_{1}^{\prime}(\zeta)\right)^{2} \cdot \frac{1}{\xi_{1}(\zeta)}-\frac{\left(2+\xi_{1}^{\prime}(\zeta)\right)^{2}}{0.092-2 \zeta-\xi_{1}(\zeta)} \\
A_{2}(\zeta) & :=\xi_{1}^{\prime \prime}(\zeta) \cdot \ln \left(0.092-2 \zeta-\xi_{1}(\zeta)\right)-\xi_{1}^{\prime \prime}(\zeta) \cdot \ln \left(\xi_{1}(\zeta)\right), \\
A_{3}(\zeta) & :=\ln \left(\frac{4}{1.618}\right) \cdot \xi_{1}^{\prime \prime}(\zeta), \\
A_{4}(\zeta) & :=\frac{4}{0.046+2 \zeta}+\frac{2}{0.408+\zeta}+\frac{18}{0.408-3 \zeta}, \text { and } \\
A_{5}(\zeta) & :=\xi_{1}^{\prime \prime}(\zeta) \cdot \ln \left(0.092+4 \zeta-\xi_{1}(\zeta)\right)-\frac{\left(\xi_{1}^{\prime}(\zeta)-4\right)^{2}}{0.092+4 \zeta-\xi_{1}(\zeta)} \\
& -\xi_{1}^{\prime \prime}(\zeta) \cdot \ln \left(1.04-5 \zeta+\xi_{1}(\zeta)\right)-\frac{\left(\xi_{1}^{\prime}(\zeta)-5\right)^{2}}{1.04-5 \zeta+\xi_{1}(\zeta)},
\end{aligned}
$$

so that (19) holds.

Claim 23. For each $\zeta \in[0,0.046), A_{1}(\zeta)<0$.

Proof: Since $0.092-2 \zeta-\xi_{1}(\zeta) \geqslant 0$ and $\xi_{1}(\zeta) \geqslant 0$, by Claim 22 ,

$$
\begin{aligned}
A_{1}(\zeta) & =\frac{1}{0.046-\zeta}-\left(\xi_{1}^{\prime}(\zeta)\right)^{2} \cdot \frac{1}{\xi_{1}(\zeta)}-\left(2+\xi_{1}^{\prime}(\zeta)\right)^{2} \cdot \frac{1}{0.092-2 \zeta-\xi_{1}(\zeta)} \\
& \leqslant \frac{1}{0.046-\zeta}-\left(\xi_{1}^{\prime}(\zeta)\right)^{2} \cdot \frac{1}{0.092-2 \zeta}-\left(2+\xi_{1}^{\prime}(\zeta)\right)^{2} \cdot \frac{1}{0.092-2 \zeta}
\end{aligned}
$$




$$
=\frac{1}{0.046-\zeta}-\frac{\left(\xi_{1}^{\prime}(\zeta)+1\right)^{2}+1}{0.046-\zeta}=-\frac{\left(\xi_{1}^{\prime}(\zeta)+1\right)^{2}}{0.046-\zeta}<0
$$

Claim 24. For each $\zeta \in[0,0.046), A_{2}(\zeta)<0$.

Proof: Let $\zeta \in[0,0.046)$. By Claim 21, inequality $A_{2}(\zeta)<0$ is equivalent to

$$
0.092-2 \zeta-\xi_{1}(\zeta)>\xi_{1}(\zeta)
$$

Let $y(\zeta)=0.092-2 \zeta-2 \xi_{1}(\zeta)$. By Claim 22,

$$
y^{\prime}(\zeta)=-2-2 \xi_{1}^{\prime}(\zeta)<0
$$

Therefore, $y(\zeta)>y(0.046)=0$ for each $\zeta \in[0,0.046)$. This proves the claim.

Claim 25. For each $\zeta \in[0,0.046), A_{3}(\zeta) \leqslant-22.46$.

Proof: This follows from the definition $(22)$, since $\xi_{1}^{\prime \prime}(\zeta) \leqslant-24.822$ by Claim 21.

Claim 26. The function $A_{4}^{\prime}(\zeta)$ has exactly one root $d_{\zeta}$ in the interval $[0,0.046]$. Furthermore, $d_{\zeta} \in(0.0355167,0.0355168)$, and $A_{4}(\zeta)$ is decreasing on $\left[0, d_{\zeta}\right]$ and increasing on $\left[d_{\zeta}, 0.046\right]$.

Proof: By Definition (23),

$$
A_{4}^{\prime}(\zeta)=-\frac{2}{(\zeta+0.023)^{2}}-\frac{2}{(\zeta+0.408)^{2}}+\frac{6}{(\zeta-0.136)^{2}}
$$

and

$$
A_{4}^{\prime \prime}(\zeta)=\frac{4}{(\zeta+0.023)^{3}}+\frac{4}{(\zeta+0.408)^{3}}-\frac{12}{(\zeta-0.136)^{3}} .
$$

The last expression is positive for all $\zeta \in[0,0.046]$, so function $A_{4}^{\prime}(\zeta)$ may have at most one root on $[0,0.046]$. On the other hand, $A_{4}^{\prime}(0.0355167)<-0.002$ and $A_{4}^{\prime}(0.0355168)>$ 0.0006. This proves the claim.

Claim 27. For each $\zeta \in[0,0.046), A_{4}(\zeta)+A_{5}(\zeta) \leqslant 20$.

Proof: Let

$$
z_{1}(\zeta)=0.092+4 \zeta-\xi_{1}(\zeta) \text { and } z_{2}(\zeta)=1.04-5 \zeta+\xi_{1}(\zeta)
$$

By Claim 22, $z_{1}^{\prime}(\zeta)=4-\xi_{1}^{\prime}(\zeta)>0$ and $z_{2}^{\prime}(\zeta)=-5+\xi_{1}^{\prime}(\zeta)<0$ for each $\zeta \in[0,0.046)$. So,

$$
z_{1}(\zeta) \text { is increasing and } z_{2}(\zeta) \text { is decreasing on }[0,0.046) \text {. }
$$

Since

$$
z_{1}(\zeta)<z_{1}(0.046)<z_{2}(0.046)<z_{2}(\zeta)
$$

for each $\zeta \in[0,0.046)$, Definitions (23) and (24) together with Claim 21 yield

$$
A_{4}(\zeta)+A_{5}(\zeta)=A_{4}(\zeta)+\xi_{1}^{\prime \prime}(\zeta) \cdot \ln \left(z_{1}(\zeta)\right)-\frac{\left(\xi_{1}^{\prime}(\zeta)-4\right)^{2}}{z_{1}(\zeta)}-\xi_{1}^{\prime \prime}(\zeta) \cdot \ln \left(z_{2}(\zeta)\right)-\frac{\left(\xi_{1}^{\prime}(\zeta)-5\right)^{2}}{z_{2}(\zeta)}
$$




$$
\begin{aligned}
& \leqslant A_{4}(\zeta)-27.336 \cdot\left(\ln \left(z_{1}(\zeta)\right)-\ln \left(z_{2}(\zeta)\right)\right)-\frac{\left(\xi_{1}^{\prime}(\zeta)-4\right)^{2}}{z_{1}(\zeta)}-\frac{\left(\xi_{1}^{\prime}(\zeta)-5\right)^{2}}{z_{2}(\zeta)} \\
& =: Q(\zeta)
\end{aligned}
$$

Since $\zeta \in[0,0.046)$, it belongs to the interval $[0.001 k, 0.001(k+1))$ for some integer $0 \leqslant k \leqslant 45$. We consider 3 cases.

Case 1: $0 \leqslant k \leqslant 34$. Then by Claim 26 and $(25)$, for each $\zeta \in[0.001 k, 0.001(k+1))$,

$$
\begin{gathered}
A_{4}(0.001 k) \geqslant A_{4}(\zeta), \\
z_{1}(\zeta) \geqslant z_{1}(0.001 k) \text { and } z_{2}(0.001 k) \geqslant z_{2}(\zeta) .
\end{gathered}
$$

Therefore,

$$
\begin{aligned}
Q(\zeta) \leqslant & M_{1}(k):=A_{4}(0.001 k)-27.336 \cdot\left(\ln \left(z_{1}(0.001 k)\right)-\ln \left(z_{2}(0.001 k)\right)\right) \\
& -\frac{\left(\xi_{1}^{\prime}(0.001 k)-4\right)^{2}}{z_{1}(0.001(k+1))}-\frac{\left(\xi_{1}^{\prime}(0.001 k)-5\right)^{2}}{z_{2}(0.001 k)}
\end{aligned}
$$

The bounds for $M_{1}(k)$ certifying that $M_{1}(k)<20$ for each $0 \leqslant k \leqslant 34$ are given in Table 2 in Appendix 1.

Case 2: $k=35$. Similarly to Case 1,

$$
\begin{aligned}
Q(\zeta) \leqslant & \max \left(A_{4}(0.035), A_{4}(0.036)\right)-27.336 \cdot\left(\ln \left(z_{1}(0.035)\right)-\ln \left(z_{2}(0.035)\right)\right) \\
& -\frac{\left(\xi_{1}^{\prime}(0.035)-4\right)^{2}}{z_{1}(0.036)}-\frac{\left(\xi_{1}^{\prime}(0.035)-5\right)^{2}}{z_{2}(0.035)} \\
& <98.404-27.336 \cdot(-1.5-(-0.135))-94-36.3<5.5<20 .
\end{aligned}
$$

Case 3: $36 \leqslant k \leqslant 45$. Again, similarly to Case 1 ,

$$
\begin{aligned}
Q(\zeta) \leqslant M_{3}(k):= & A_{4}(0.001(k+1))-27.336 \cdot\left(\ln \left(z_{1}(0.001 k)\right)-\ln \left(z_{2}(0.001 k)\right)\right) \\
& -\frac{\left(\xi_{1}^{\prime}(0.001 k)-4\right)^{2}}{z_{1}(0.001(k+1))}-\frac{\left(\xi_{1}^{\prime}(0.001 k)-5\right)^{2}}{z_{2}(0.001 k)} .
\end{aligned}
$$

The bounds for $M_{1}(k)$ certifying that $M_{1}(k)<20$ for each $36 \leqslant k \leqslant 45$ are given in Table 1 in Appendix 1.

Thus by (19) and Claims 23-27, for each $\zeta \in[0,0.046)$,

$$
\frac{\partial^{2} \ln \left(h_{1}(0.454, \zeta)\right)}{\partial \zeta^{2}}=\sum_{i=1}^{5} A_{i}(\zeta)<-22.46+20=-2.46<0 .
$$

We also can check by plugging in the values that

$$
\frac{\partial \ln \left(h_{1}(0.454,0.0228718)\right)}{\partial \zeta}>7.54 \cdot 10^{-8}, \text { and } \frac{\partial \ln \left(h_{1}(0.454,0.0228719)\right)}{\partial \zeta}<-9 \cdot 10^{-6} .
$$

Thus, the derivative of $h_{1}(0.454, \zeta)$ equals 0 at a unique $\zeta_{1} \in(0.0228718,0.0228719)$. 
Recall that $h_{1}(0.454, \zeta)>0$ for $\zeta \in[0,0.046)$. Therefore, after comparing the value $h_{1}(0.454,0.0228719)$ with the boundary values $h_{1}(0.454,0)$ and $h_{1}(0.454,0.46)$, we conclude that the maximum of $h_{1}(0.454, \zeta)$ is attained at $\zeta_{1}$. We can plug in numbers into a computer and obtain that

$$
\begin{aligned}
h_{1}(0.454,0.0228718) & \leqslant 0.999982, \\
\frac{\partial \ln \left(h_{1}(0.454,0.0228718)\right)}{\partial \zeta} & \leqslant 1 \cdot 10^{-7},
\end{aligned}
$$

and

$$
\frac{\partial h_{1}(0.454,0.0228718)}{\partial \zeta}=h_{1}(0.454,0.0228718) \cdot \frac{\partial \ln \left(h_{1}(0.454,0.0228718)\right)}{\partial \zeta} \leqslant 1 \cdot 10^{-7},
$$

which implies that

$$
h_{1}\left(0.454, \zeta_{1}\right) \leqslant h_{1}(0.454,0.0228718)+1 \cdot 10^{-7} \cdot 0.0000001 \leqslant 0.999983 .
$$

The proof of the next lemma is similar but significantly simpler. It is mostly a routine bounding some expressions. So, we present the proof of Lemma 28 in Appendix 2.

Lemma 28. For every

$$
(\chi, \zeta, \xi) \in \Omega=\left\{(\chi, \zeta, \xi): 0.454<\chi \leqslant 0.45537,0 \leqslant \zeta \leqslant \frac{1}{2}-\chi, 0 \leqslant \xi \leqslant 1-2 \chi-2 \zeta\right\},
$$

we have

$$
\frac{\partial \ln (h(\chi, \zeta, \xi))}{\partial \chi}<0
$$

Since $h(\chi, \zeta, \xi)>0$ for each $(\chi, \zeta, \xi) \in \Omega$, Lemma 28 yields that for each fixed $\zeta$ and $\xi$, the maximum of $h(\chi, \zeta, \xi)$ over $(\chi, \zeta, \xi) \in \Omega$ is attained at $\chi=0.454$. By Lemma 20, this maximum is at most 0.999983 . This yields (14).

\section{Completion of the proof of Theorem 17}

By (14) and Lemma 19, for all positive integers $n$ and $x$ such that $n$ is even and $0.454 n<$ $x \leqslant 0.45537 n$,

$$
\frac{q(x, n)}{(3 n-1) ! !} \leqslant O\left(n^{6}\right) \cdot 0.999983^{n}
$$

It follows that

$$
\frac{1}{(3 n-1) ! !} \sum_{x=\lceil 0.454 n\rceil}^{\lfloor 0.45537 n\rfloor} q(x, n) \leqslant O\left(n^{7}\right) \cdot 0.999983^{n} \rightarrow 0 \quad \text { as } n \rightarrow \infty .
$$

Thus by Lemma 18, the number of pairings $F \in \mathcal{G}_{16}^{\prime}(n)$ with $0.454 n<\alpha(F) \leqslant 0.45537 n$ is $o((3 n-1) ! !)$. Together with Theorem 16, this means that almost no pairings have independence ratio larger than 0.454 . Thus by Corollary 5 we conclude that almost no $n$-vertex 3-regular graphs of girth at least 16 have independence ratio larger than 0.454 . This proves Theorem 17 and thus also Theorem 2 .

Acknowledgment. We thank Jan Volec for helpful discussion and bringing [4] to our attention. We thank a referee for the valuable comments. 


\section{References}

[1] E.A. Bender and E.R. Canfield, The asymptotic number of non-negative integer matrices with given row and column sums, Journal of Combinatorial Theory A, 24 (1978), 296-307.

[2] B. Bollobás, The independence ratio of regular graphs, Proc. Amer. Math. Soc. 83 (1981), 433-436.

[3] B. Bollobás, A probabilistic proof of an asymptotic formula for the number of regular graphs, European J. Combinatorics 1 (1980), 311-316.

[4] E. Csóka, Independent sets and cuts in large-girth regular graphs, arXiv: 1602.02747.

[5] E. Csóka, B. Gerencsér, V. Harangi and B. Virág, Invariant Gaussian processes and independent sets on regular graphs of large girth, Random Structures and Algorithms, 47 (2015), 284-303.

[6] G. Hopkins and W. Staton, Girth and independence ratio, Canad. Math. Bull., 25 (1982), no. 2, 179-186.

[7] C. Hoppen, Properties of graphs with large girth, Doctoral thesis, University of Waterloo, 2008.

[8] C. Hoppen and N. Wormald, Properties of regular graphs with large girth via local algorithms, J. Combin. Theory Ser. B, 121 (2016), 367-397.

[9] S. Janson, T. Łuczak and A. Rucinski, Random Graphs, Interscience Series in Discrete Mathematics and Optimization, John Wiley, 2000.

[10] F. Kardoš, D. Král and J. Volec, Fractional colorings of cubic graphs with large girth, SIAM J. Discrete Math. 25 (2011), 1454-1476.

[11] J. Lauer and N. Wormald, Large independent sets in regular graphs of large girth, J. Combin. Theory Ser. B, 97 (2007), 999-1009.

[12] B. D. McKay, Independent sets in regular graphs of high girth. Proceedings of the Singapore conference on combinatorial mathematics and computing (Singapore, 1986). Ars Combin. 23 (1987), A, 179-185.

[13] J. Shearer, A note on the independence number of triangle-free graphs, II, J. Combin. Theory Ser. B, 53 (1991), 300-307.

[14] N. C. Wormald, Some problems in the enumeration of labelled graphs, Doctoral Thesis, Univ. of Newcastle (1978). 


\section{Appendix 1: Tables for Claim 27}

We define the following functions for the table.

\begin{tabular}{|c|c|c|c|c|c|c|}
\hline \multicolumn{5}{|c|}{$T_{4}(k):=-\frac{\left(\xi_{1}^{\prime}(0.001 k)-4\right)^{2}}{z_{1}(0.001(k+1))}$} & \multicolumn{2}{|c|}{$\frac{\left(\xi_{1}^{\prime}(0.001 k)-5\right)^{2}}{z_{2}(0.001 k)}}$. \\
\hline$k$ & $T_{1}(k)$ & $T_{2}(k)$ & $T_{3}(k)$ & $T_{4}(k)$ & $T_{5}(k)$ & $M_{1}(k)$ \\
\hline 0 & 135.9762 & 2.553562 & 0.05277836 & -166.7356 & -20.83335 & 19.7 \\
\hline 1 & 132.6679 & 2.507105 & 0.04831009 & -161.790 & -21.1686 & 19.6 \\
\hline 2 & 129.6543 & 2.462392 & 0.04379588 & -157.2333 & -21.50947 & 19.5 \\
\hline 3 & 126.903 & 2.419288 & 0.03923514 & -153.0194 & -21.85574 & 19.3 \\
\hline 4 & 124.384 & 2.377674 & 0.03462729 & -149.1122 & -22.20758 & 19.1 \\
\hline 5 & 122.0728 & 2.33745 & 0.02997174 & -145.4794 & -22.56505 & 18.8 \\
\hline 6 & 119.9504 & 2.298492 & 0.02526786 & -142.0935 & -22.92824 & 18.5 \\
\hline 7 & 117.9977 & 2.260743 & 0.02051507 & -138.9302 & -23.29722 & 18.2 \\
\hline 8 & 116.1989 & 2.224115 & 0.01571273 & -135.9683 & -23.67205 & 17.8 \\
\hline 9 & 114.5404 & 2.188538 & 0.01086022 & -133.1892 & -24.05282 & 17.5 \\
\hline 10 & 113.0099 & 2.153948 & 0.005956888 & -130.5765 & -24.43961 & 17.1 \\
\hline 11 & 111.5969 & 2.120286 & 0.001002109 & -128.1157 & -24.83248 & 16.7 \\
\hline 12 & 110.292 & 2.0876 & -0.004004782 & -125.793 & -25.23152 & 16.3 \\
\hline 13 & 109.0867 & 2.055542 & -0.009064451 & -123.5994 & -25.63682 & 15.8 \\
\hline 14 & 107.9738 & 2.024367 & -0.01417756 & -121.5220 & -26.04845 & 15.4 \\
\hline 15 & 106.9466 & 1.993934 & -0.01934483 & -119.5525 & -26.4664 & 15.0 \\
\hline 16 & 106.000 & 1.964204 & -0.02456692 & -117.6823 & -26.89104 & 14.5 \\
\hline 17 & 105.1262 & 1.935144 & -0.02984456 & -115.9041 & -27.32218 & 14.0 \\
\hline 18 & 104.3229 & 1.90673 & -0.03517846 & -114.2111 & -27.76000 & 13.6 \\
\hline 19 & 103.585 & 1.878905 & -0.04056935 & -112.5970 & -28.20460 & 13.1 \\
\hline 20 & 102.9088 & 1.851668 & -0.04601797 & -111.0562 & -28.65606 & 12.6 \\
\hline 21 & 102.2906 & 1.824985 & -0.05152507 & -109.5836 & -29.1144 & 12.1 \\
\hline 22 & 101.7273 & 1.798832 & -0.05709143 & -108.1746 & -29.57998 & 11.6 \\
\hline 23 & 101.217 & 1.773185 & -0.06271781 & -106.8248 & -30.05263 & 11.1 \\
\hline 24 & 100.7543 & 1.748024 & -0.06840502 & -105.5304 & -30.53255 & 10.7 \\
\hline 25 & 100.3398 & 1.723329 & -0.07415386 & -104.2877 & -31.01984 & 10.2 \\
\hline 26 & 99.97009 & 1.699082 & -0.07996514 & -103.0934 & -31.51462 & 9.7 \\
\hline 27 & 99.64358 & 1.675265 & -0.08583972 & -101.9446 & -32.016 & 9.2 \\
\hline 28 & 99.3585 & 1.651862 & -0.09177843 & -100.8383 & -32.52708 & 8.7 \\
\hline 29 & 99.11297 & 1.628858 & -0.09778215 & -99.77206 & -33.04501 & 8.2 \\
\hline 30 & 98.90584 & 1.606239 & -0.1038517 & -98.74333 & -33.5708 & 7.7 \\
\hline 31 & 98.7358 & 1.583989 & -0.1099881 & -97.74996 & -34.10486 & 7.2 \\
\hline 32 & 98.6015 & 1.562098 & -0.1161922 & -96.78986 & -34.64704 & 6.7 \\
\hline 33 & 98.50187 & 1.54056 & -0.122464 & -95.86113 & -35.19758 & 6.3 \\
\hline 34 & 98.43615 & 1.519339 & -0.1288073 & -94.96198 & -35.75661 & 5.8 \\
\hline
\end{tabular}

Table 1: Upper bounds for expressions in $M_{1}(k)$. 


\begin{tabular}{c|c|c|c|c|c|c|}
\hline$k$ & $T_{1}(k+1)$ & $T_{2}(k)$ & $T_{3}(k)$ & $T_{4}(k)$ & $T_{5}(k)$ & $M_{3}(k)$ \\
\hline 36 & 98.43379 & 1.477873 & -0.1417047 & -93.24588 & -36.90074 & 4.9 \\
\hline 37 & 98.49569 & 1.457599 & -0.1482617 & -92.42593 & -37.48615 & 4.4 \\
\hline 38 & 98.58802 & 1.437619 & -0.1548924 & -91.62957 & -38.08066 & 4.0 \\
\hline 39 & 98.71033 & 1.417923 & -0.1615978 & -90.85551 & -38.68445 & 3.6 \\
\hline 40 & 98.86225 & 1.398503 & -0.1683790 & -90.1025 & -39.29768 & 3.1 \\
\hline 41 & 99.04347 & 1.379352 & -0.1752370 & -89.36971 & -39.92054 & 2.7 \\
\hline 42 & 99.25376 & 1.36046 & -0.1821731 & -88.65582 & -40.55321 & 2.3 \\
\hline 43 & 99.49293 & 1.341822 & -0.1891883 & -87.95995 & -41.1958 & 1.9 \\
\hline 44 & 99.76085 & 1.323429 & -0.1962838 & -87.28121 & -41.84877 & 1.5 \\
\hline 45 & 100.0576 & 1.305276 & -0.2034610 & -86.61873 & -42.51206 & 1.1 \\
\hline
\end{tabular}

Table 2: Upper bounds for expressions in $M_{3}(k)$.

\section{Appendix 2: Proof of Lemma 28}

By definition, the boundary, $\partial \Omega$, of $\Omega$ is

$$
\begin{aligned}
\partial \Omega= & \{(\chi, \zeta, \xi): \xi=0,2 \chi+2 \zeta \leqslant 1,0.454 \leqslant \chi \leqslant 0.45537, \zeta \geqslant 0\} \cup \\
& \{(\chi, \zeta, \xi): \zeta=0,2 \chi+\xi \leqslant 1,0.454 \leqslant \chi \leqslant 0.45537, \xi \geqslant 0\} \cup \\
& \{(\chi, \zeta, \xi): \chi=0.454,2 \zeta+\xi \leqslant 0.092, \zeta \geqslant 0, \xi \geqslant 0\} \cup \\
& \{(\chi, \zeta, \xi): \chi=0.45537,2 \zeta+\xi \leqslant 0.08926, \zeta \geqslant 0, \xi \geqslant 0\} .
\end{aligned}
$$

We also will consider the 2-dimensional set

$$
\Omega_{1}=\{(\chi, \zeta): 0.454 \leqslant \chi \leqslant 0.45537,0 \leqslant \zeta \leqslant 0.5-\chi\} .
$$

Then the boundary of $\Omega_{1}$ is

$$
\begin{aligned}
\partial \Omega_{1}= & \{(\chi, \zeta): 0.454 \leqslant \chi \leqslant 0.45537, \zeta=0\} \cup\{(\chi, \zeta): 0 \leqslant \zeta \leqslant 0.046, \chi=0.454\} \\
& \cup\{(\chi, \zeta): 0 \leqslant \zeta \leqslant 0.04463, \chi=0.45537\} \cup\left\{(\chi, \zeta): 0.454 \leqslant \chi \leqslant 0.45537, \chi+\zeta=\frac{1}{2}\right\} .
\end{aligned}
$$

By the definition of $h$,

$$
\begin{aligned}
\frac{\partial \ln (h(\chi, \zeta, \xi))}{\partial \chi}= & 4 \ln \left(2 \chi-3 \zeta-\frac{1}{2}\right)+4 \ln \left(2 \chi+\zeta-\frac{1}{2}\right)-\ln (1-2 \chi-2 \zeta) \\
& -\ln (1-2 \chi+4 \zeta)+2 \ln (1-2 \chi-2 \zeta-\xi)+2 \ln (1-2 \chi+4 \zeta-\xi) \\
& -10 \ln \left(10 \chi-5 \zeta+\xi-\frac{7}{2}\right) .
\end{aligned}
$$

Similarly to the proof of Lemma 20, we present $\frac{\partial \ln (h(\chi, \zeta, \xi))}{\partial \chi}$ in the form $\sum_{j=1}^{6} B_{j}$, where

$$
\begin{aligned}
B_{1}(\chi, \zeta) & :=4 \ln \left(2 \chi-3 \zeta-\frac{1}{2}\right), \quad B_{2}(\chi, \zeta):=4 \ln \left(2 \chi+\zeta-\frac{1}{2}\right), \\
B_{3}(\chi, \zeta, \xi) & :=2 \ln (1-2 \chi-2 \zeta-\xi)-\ln (1-2 \chi-2 \zeta), \quad B_{4}(\chi, \zeta):=\ln (1-2 \chi+4 \zeta), \\
B_{5}(\chi, \zeta, \xi) & :=2 \ln (1-2 \chi+4 \zeta-\xi), \quad \text { and } B_{6}(\chi, \zeta, \xi):=\ln \left(10 \chi-5 \zeta+\xi-\frac{7}{2}\right),
\end{aligned}
$$

and then bound each of the terms separately. 
Claim 29. For all $(\chi, \zeta) \in \Omega_{1}, B_{1}(\chi, \zeta)<-3.55$.

Proof: For each $(\chi, \zeta) \in \Omega_{1}$, we have $\chi-\frac{3}{2} \zeta-0.25>0$, since $\chi \geqslant 0.454$ and $\zeta \leqslant 0.046$. As for each $(\chi, \zeta) \in \Omega_{1}$,

$$
\frac{\partial B_{1}(\chi, \zeta)}{\partial \chi}=\frac{4}{\chi-\frac{3}{2} \zeta-0.25}>0 \quad \text { and } \quad \frac{\partial B_{1}(\chi, \zeta)}{\partial \zeta}=\frac{-6}{\chi-\frac{3}{2} \zeta-0.25}<0,
$$

the maximum is attained at a corner on the boundary $\partial \Omega_{1}$. Comparing the values of $B_{1}$ at the four corners of $\partial \Omega_{1}$, we see that the maximum is attained at $(\chi, \zeta)=(0.45537,0)$ and $B_{1}(0.45537,0)<-3.55$.

Claim 30. For all $(\chi, \zeta) \in \Omega_{1}, B_{2}(\chi, \zeta)<-3.14$.

Proof: For each $(\chi, \zeta) \in \Omega_{1}$, we have $2 \chi+\zeta-\frac{1}{2}>0$. As for each $(\chi, \zeta) \in \Omega_{1}$,

$$
\frac{\partial B_{2}(\chi, \zeta)}{\partial \chi}=\frac{8}{2 \chi+\zeta-\frac{1}{2}}>0, \quad \text { and } \quad \frac{\partial B_{2}(\chi, \zeta)}{\partial \zeta}=4 \cdot(2 \chi+\zeta-0.5)^{-1}>0,
$$

the maximum is attained at a corner of the boundary $\partial \Omega_{1}$. Comparing the values of $B_{2}$ at the four corners of $\partial \Omega_{1}$, we see that the maximum is attained at $(\chi, \zeta)=$ $(0.45537,0.04463)$, and $B_{2}(0.45537,0.04463)<-3.14$.

Claim 31. For all $(\chi, \zeta, \xi) \in \Omega, B_{3}(\chi, \zeta, \xi)<0$.

Proof: We can write $B_{3}(\chi, \zeta, \xi)$ in the form

$$
B_{3}(\chi, \zeta, \xi)=\ln (1-2 \chi-2 \zeta-\xi)+\ln \left(\frac{1-2 \chi-2 \zeta-\xi}{1-2 \chi-2 \zeta}\right)
$$

and observe that $\ln (1-2 \chi-2 \zeta-\xi)<0($ since $2 \chi+2 \zeta+\xi>0)$ and $\ln \left(\frac{1-2 \chi-2 \zeta-\xi}{1-2 \chi-2 \zeta}\right) \leqslant 0$ (since $1-2 \chi-2 \zeta-\xi \leqslant 1-2 \chi-2 \zeta$ ).

Claim 32. For all $(\chi, \zeta) \in \Omega_{1}, B_{4}(\chi, \zeta)<-1.28$.

Proof: For each $(\chi, \zeta) \in \Omega_{1},-2 \chi+4 \zeta+1>0$. As for each $(\chi, \zeta) \in \Omega_{1}$,

$$
\frac{\partial B_{4}(\chi, \zeta)}{\partial \chi}=\frac{-2}{-2 \chi+4 \zeta+1}<0, \quad \text { and } \quad \frac{\partial B_{4}(\chi, \zeta)}{\partial \zeta}=\frac{4}{-2 \chi+4 \zeta+1}>0,
$$

the maximum of $B_{4}$ is attained at a corner of the boundary $\partial \Omega_{1}$. Comparing the values of $B_{4}$ at the four corners of $\partial \Omega_{1}$, we see that the maximum is attained at $(\chi, \zeta)=$ $(0.454,0.046)$, and $B_{4}(0.454,0.046)<-1.28$.

Claim 33. For all $(\chi, \zeta, \xi) \in \Omega, B_{5}(\chi, \zeta, \xi)<-2.57$.

Proof: For each $(\chi, \zeta, \xi) \in \Omega-\partial \Omega$, we have $2 \chi-4 \zeta+\xi-1<0$ since $2 \chi+2 \zeta+\xi<1 \leqslant$ $1+4 \zeta+2 \zeta$. Since

$$
\lim _{2 \chi+\xi \rightarrow 1} B_{5}(\chi, 0, \xi)=-\infty,
$$

the maximum of $B_{5}$ is not attained at $\zeta=0,2 \chi+\xi=1$. As for each $(\chi, \zeta, \xi) \in \Omega$,

$$
\frac{\partial B_{5}(\chi, \zeta, \xi)}{\partial \chi}=\frac{4}{2 \chi-4 \zeta+\xi-1}<0, \quad \frac{\partial B_{5}(\chi, \zeta, \xi)}{\partial \zeta}=\frac{-8}{2 \chi-4 \zeta+\xi-1}>0,
$$


and

$$
\frac{\partial B_{5}(\chi, \zeta, \xi)}{\partial \xi}=\frac{2}{2 \chi-4 \zeta+\xi-1}<0
$$

the maximum of $B_{5}$ is attained at a corner of the boundary $\partial \Omega$. Comparing the values of $B_{5}$ at the corners of $\partial \Omega$, we see that the maximum is attained at $(\chi, \zeta, \xi)=$ $(0.454,0.046,0)$ and $B_{5}(0.454,0.046,0)<-2.57$.

Claim 34. For all $(\chi, \zeta, \xi) \in \Omega, B_{6}(\chi, \zeta, \xi)<0.14$.

Proof: For each $(\chi, \zeta, \xi) \in \Omega$, we have $10 \chi-5 \zeta+\xi-\frac{7}{2}>0$ since $10 \chi-\frac{7}{2} \geqslant 1.04$ and $5 \zeta \leqslant 0.23$. As for each $(\chi, \zeta, \xi) \in \Omega$,

$$
\frac{\partial B_{6}(\chi, \zeta, \xi)}{\partial \chi}=\frac{10}{10 \chi-5 \zeta+\xi-\frac{7}{2}}>0, \quad \frac{\partial B_{6}(\chi, \zeta, \xi)}{\partial \zeta}=\frac{-5}{10 \chi-5 \zeta+\xi-\frac{7}{2}}<0,
$$

and

$$
\frac{\partial B_{6}(\chi, \zeta, \xi)}{\partial \xi}=\frac{1}{10 \chi-5 \zeta+\xi-\frac{7}{2}}>0
$$

the maximum of $B_{5}$ is attained at a corner of the boundary $\partial \Omega$. Comparing the values of $B_{6}$ at the corners of $\partial \Omega$, we see that the maximum is attained at $(\chi, \zeta, \xi)=$ $(0.45537,0,0.08926)$ and $B_{6}(0.454,0,0.08926)<0.14$.

By Claims 29-34, for each $(\chi, \zeta, \xi) \in \Omega$,

$$
\begin{aligned}
\frac{\partial \ln (h(\chi, \zeta, \xi))}{\partial \chi} & =B_{1}(\chi, \zeta)+B_{2}(\chi, \zeta)+B_{3}(\chi, \zeta, \xi)+B_{4}(\chi, \zeta)+B_{5}(\chi, \zeta, \xi)+B_{6}(\chi, \zeta, \xi) \\
& <-3.55-3.14+0-1.28-2.57+0.14<0
\end{aligned}
$$

